\title{
TOTALLY DISCONNECTED JULIA SET FOR DIFFERENT CLASSES OF MEROMORPHIC FUNCTIONS
}

\author{
P. DOMÍNGUEZ, A. HERNÁNDEZ, AND G. SIENRA \\ In Memory of I. N. Baker
}

\begin{abstract}
We study a class of functions $\mathbf{A}$ given by Epstein in Towers of $\mathrm{fi}$ nite type complex analytic maps, ProQuest LLC, Ann Arbor, MI, 1993, called finite-type maps. We extend a result related with the Julia set given by Baker, Domínguez in Some connectedness properties of Julia sets, Complex Variable Theory Appl. 41 (2000), 371-389, and Baker, Domínguez, and Herring in Dynamics of functions meromorphic outside a small set, Ergodic Theory Dynam. Systems 21 (2001), 647-672, to functions in class A.
\end{abstract}

\section{INTRODUCTION}

Let $X, Y$ be compact Riemann surfaces (complex 1-manifolds) and let $D_{f}$ be an arbitrary non-empty open subset of $X$. We define

$$
\begin{gathered}
\operatorname{Hol}(X, Y)=\left\{f: D_{f} \rightarrow Y \mid f \text { is analytic }\right\}, \\
\operatorname{Hol}(X, X)=\operatorname{Hol}(X) .
\end{gathered}
$$

In 14,15] Epstein defines and studies a class of functions called finite-type maps; we will denote this class by $\mathbf{A}$ and the definition is as follows.

Definition 1.1. $f$ is in class $\mathbf{A}$ (finite-type maps) if $f \in H o l(X)$ with the following properties:

(a) $f$ is nowhere constant (not constant on any component of $D_{f} \subset X$ ).

(b) There are no isolated removable singularities, so every isolated singularity is essential.

(c) The set of singular values of $f$ is finite.

The set of singular values of $f \in \operatorname{Hol}(X, Y)$ is $S V(f)=\overline{C(f) \cup A(f)}$, where $C(f)$ is the set of critical values and $A(f)$ is the set of asymptotic values.

The closure of the post-singular set of $f \in \operatorname{Hol}(X, Y)$ is denoted by $P S V(f)$, where the post-singular set is the smallest forward invariant set containing $S V(f)$.

For functions in class $\mathbf{A}$ the identity map on $X$ is $I_{X}: X \rightarrow X$ and the iterate $f^{n+1}=f \circ \cdots \circ f, n \geq 1$, has domain of definition $D_{f^{n+1}}=f^{-1}\left(D_{f^{n}}\right) \cap D_{f^{n}}$, for $n \geq 1$, where $f^{0}=I_{X}$.

We denote the set $D_{\infty}$ as the interior of $\bigcap_{n=1}^{\infty} D_{f^{n}}$ which is the domain of the iterates.

Received by the editors September 24, 2012.

2010 Mathematics Subject Classification. Primary 37F10; Secondary 30D05.

The authors were supported by CONACYT projects numbers 128005 and 153850 .

(C) 2014 American Mathematical Society Reverts to public domain 28 years from publication 
Definition 1.2. Given $f \in \operatorname{Hol}(X), f$ nowhere constant, $z \in X$ is said to be normal if $z \in X \backslash \overline{D_{\infty}}$ or if it has a neighbourhood $U$ such that $U \subset D_{\infty}$ and $\left\{\left.f^{n}\right|_{U}\right\}$ is a normal family.

The Fatou set $F_{X}(f)$ for functions in class $\mathbf{A}$ is the set of normal points. The complement is called the Julia set $J_{X}(f)$. We will denote by $F(z)=F_{X}(f)$ and by $J(f)=J_{X}(f)$.

In [14, 15] Epstein gives the following definition for functions $f \in \operatorname{Hol}(T, X)$, where $f$ is nowhere constant (not constant on any component of $D_{f}$ ).

Definition 1.3. An analytic map on a Riemann surface $X$ is typical if $D_{\infty}$ is hyperbolic, exceptional otherwise.

Examples of exceptional maps are the rational maps, entire maps and selfanalytic maps of $\mathbb{C} \backslash\{0\}$.

Remark 1.4. In 14 Epstein proves that for functions in class $\mathbf{A}$ we have

(a) every periodic component of the Fatou set is a super-attracting domain, attracting domain, parabolic domain, Siegel disc, or Herman ring.

(b) The boundary of any Siegel disc or Herman ring lies in $P S V(f)$.

(c) A fixed attracting or parabolic basin contains a singular value of $f$.

There are classes of meromorphic functions which belong to the class $\mathbf{A}$ for which the set of singular values is finite. Before we give these classes of functions we recall the following classes of meromorphic functions.

$\mathcal{E}=\{f: \mathbb{C} \rightarrow \mathbb{C} \mid f$ is transcendental entire $\}$.

$\mathcal{M}=\{f: \mathbb{C} \rightarrow \widehat{\mathbb{C}} \mid f$ is transcendental meromorphic and it has at least one pole which is not omitted $\}$.

$\mathcal{K}=\{f: \widehat{\mathbb{C}} \backslash B \rightarrow \widehat{\mathbb{C}} \mid B$ is a compact countable set and $f$ is meromorphic $\}$.

The set $B$ is formed by the essential singularities of $f$, where $f$ is non-constant; we assume $B$ to have at least two elements and we allow $f$ to have poles.

$\mathbf{M}=\left\{f: \widehat{\mathbb{C}} \backslash E=E^{c} \rightarrow \widehat{\mathbb{C}} \mid E\right.$ is a compact totally disconnected set and $f$ is meromorphic in $E^{c}$ with $C\left(f, D_{f}, z_{0}\right)=\widehat{\mathbb{C}}$ for all $\left.z_{0} \in E\right\}$.

The set $E$ is formed by the essential singularities of $f$. If $E=\emptyset$ we make the further assumption that $f$ is neither constant nor univalent in $\widehat{\mathbb{C}}$.

We recall that if $f$ is a function in class $\mathcal{E}, \mathcal{M}, \mathcal{K}$ or $\mathbf{M}$, the sequence formed by its iterates is denoted by $f^{0}:=\operatorname{Id}, f^{n}:=f \circ f^{n-1}, n \in \mathbb{N}$. The Fatou set $F(f)$ is defined by the set of points $z \in \mathbb{C}(z \in \widehat{\mathbb{C}} \backslash B$ for functions in class $\mathcal{K}$ and $z \in \widehat{\mathbb{C}} \backslash E$ for functions in class $\mathbf{M}$ ) such that the sequence $\left(f^{n}\right)_{n \in \mathbb{N}}$ is well defined and normal in some neighborhood of $z$. The Julia set $J(f)$ is the complement of $F(f)$.

The classes of functions above were introduced and studied by Fatou [17] and Baker [1 for class $\mathcal{E}$, by Baker, Yi Niàn and Kotus 2 5. for class $\mathcal{M}$, by Bolsch 8 10 for class $\mathcal{K}$ and by Herring [16 for class $\mathbf{M}$.

Following the idea of Erëmenko and Lyubich [12] we give the following definition.

Definition 1.5. A function $f$ in either $\mathcal{E}, \mathcal{M}, \mathcal{K}$ or $\mathbf{M}$ belongs to the class $S_{f}$ if the set of singular values of $f$ is finite (for functions in class $\mathbf{M}$ we add the condition that the linear measure of $E$ is zero). We denote $S_{\mathcal{E}}, S_{\mathcal{M}}, S_{\mathcal{K}}$ and $S_{\mathbf{M}}$ for the different classes of functions $\mathcal{E}, \mathcal{M}, \mathcal{K}$ and $\mathbf{M}$, respectively. 
Examples of functions in classes $\mathcal{E} \cap S_{\mathcal{E}}$ and $\mathcal{M} \cap S_{\mathcal{M}}$ are the families $f_{\lambda}(z)=$ $\lambda \sin z$ and $f_{\lambda}(z)=\lambda \tan z$, respectively.

An example for functions in class $\mathcal{K} \cap S_{\mathcal{K}}$ is the family $f_{\lambda}(z)=\lambda e^{\frac{1}{z^{2}-1}}-\lambda e^{-1}$, where \pm 1 are the essential singularities of $f_{\lambda}, 0$ is a fixed point of $f$, and the set of singular values is finite.

Observe that functions in $\mathcal{E} \cap S_{\mathcal{E}}, \mathcal{M} \cap S_{\mathcal{M}}, \mathcal{K} \cap S_{\mathcal{K}}$ and $\mathbf{M} \cap S_{\mathbf{M}}$ belong to the class $\mathbf{A}$, since these functions have a finite set of singular values.

In Section 2 we mention some examples of meromorphic functions for which the set of singular values is finite and the Julia set is totally disconnected. In particular for these examples there are no Devaney hairs; see [19] for a definition.

In Section 3 we prove Theorem A which generalize a result given by Baker, Domínguez and Herring in [7].

Theorem A. Let $f$ be in class $\mathbf{A}$, where $f$ is typical, and suppose that there is an attracting fixed point whose Fatou component $H$ contains all the singular values of $f$. Then $J(f)$ is totally disconnected.

As a corollary we have the same result for functions in classes $\mathcal{M} \cap S_{\mathcal{M}}$ and $\mathcal{K} \cap S_{\mathcal{K}}$

Remark 1.6. If $f$ is typical and the Julia set is totally disconnected, then the Fatou set is just one completely invariant component.

\section{Some examples of functions with totally disconnected Julia Set}

For functions in class $\mathcal{E}$ the Julia set cannot be totally disconnected, but for functions in classes $\mathcal{M}$ and $\mathcal{K}$ it is possible to have examples where the Julia set can be totally disconnected.

Example 1. In 2] it was proved that there are functions in class $\mathcal{M}$ for which $J(f) \subset \mathbb{R}$ for such $f$ and we have: (i) $J(f)=\mathbb{R}$ or $J(f)$ is a Cantor subset of $\mathbb{R}$.

The family $f_{\lambda}(z)=\lambda \tan z$ is in class $\mathcal{M} \cap S_{\mathcal{M}}$ and for $0<\lambda<1$ the family has two Picard exceptional values $\pm \lambda i$, which are in the Fatou set, and has a single attracting fixed point in $z=0$. The Julia set $J\left(f_{\lambda}\right)$ is a Cantor set and $J\left(f_{\lambda}\right) \subset \mathbb{R} \cup\{\infty\}$; see [18. The Fatou set consists of only one completely invariant component $U$, which is multiply connected; the asymptotic values $\pm \lambda i$ are in $U$.

Example 2. The family $f_{\lambda, \mu}(z)=\tan (\lambda \tan (\mu z))$ is in class $\mathcal{K} \cap S_{\mathcal{K}}$. When $|\lambda \mu|<1$ the point $z=0$ is an attracting fixed point, since $\left|f_{\lambda, \mu}^{\prime}(0)\right|=|\lambda \mu|$. The asymptotic values of $f_{\lambda, \mu}(z)$ are $\pm \lambda i$ and $\pm \tan (\lambda i)$. The family $f_{\lambda, \mu}(z)$ has totally disconnected Julia set for $\lambda, \mu$ small constants.

\section{Proof of Theorem A}

We recall that a function $f$, in either $\mathcal{E}, \mathcal{M}, \mathcal{K}$ or $\mathbf{M}$, belongs to the class $S_{f}$ if the set of singular values of $f$ is finite. We denote $S_{\mathcal{E}}, S_{\mathcal{M}}, S_{\mathcal{K}}$ and $S_{\mathcal{K}}$ for the different classes of functions $\mathcal{E}, \mathcal{M}, \mathcal{K}$ and $\mathbf{M}$, respectively.

It is known that for functions in classes $\mathcal{E} \cap S_{\mathcal{E}}$ and $\mathcal{M} \cap S_{\mathcal{M}}$ there are neither wandering domains nor Baker domains; see [11, 13] and [5].

For functions in class $\mathbf{M} \cap S_{\mathbf{M}}$ there are neither wandering domains nor Baker domains [7. Since the proofs remain valid for functions in class $\mathcal{K} \cap S_{\mathcal{K}}$ we do not prove them. For functions in class $\mathbf{A}$ the following theorem was given by Epstein in [14. 
Theorem 3.1. If $f \in \mathbf{A}$, then it has no Baker domains nor wandering domains.

For functions in class $\mathcal{E} \cap S_{\mathcal{E}}$ we must recall that: $\infty$ is an exceptional value which is in the Julia set and it is a singular value of $f$. Thus functions in class $\mathcal{E} \cap S_{\mathcal{E}}$ do not satisfy the conditions of Theorem $\mathrm{A}$, since $f$ is exceptional. Indeed Theorem A cannot be true for functions in class $\mathcal{E} \cap S_{\mathcal{E}}$ by the arguments that we explain at the end of (ii) in the proof.

We follow and verfy that the proof given in [7] for functions in class $\mathbf{M} \cap S_{\mathbf{M}}$ works with some changes for functions in class $\mathbf{A}$, where $f$ is typical.

Proof of Theorem A. Let us assume that $f \in \mathbf{A}$ and that zero is an attracting fixed point of $f$ which belongs to the component $H$ of $F(f)$. We denote by $\left\{a_{1}, a_{2}, \ldots, a_{p}\right\}$ the set of singular values of $f$; this set is finite and $\left\{a_{1}, a_{2}, \ldots, a_{p}\right\} \subset H$. Thus $f(H) \subset H$.

(i) First we will show that the component $H$ is completely invariant and it is the only one.

Take $z$ in $H$ such that $z_{1}=f(z)$ is different from $a_{j}, 1 \leq j \leq p$, and denote by $g$ the branch of $f^{-1}$ such that $g\left(z_{1}\right)=z$. If $h$ is an arbitrary branch of $f^{-1}$, defined and hence analytic in a neighborhood of $z_{1}$, there is some closed path $\gamma$ in $\Omega=\widehat{\mathbb{C}} \backslash\left\{a_{1}, \ldots, a_{p}\right\}$ starting and ending at $z_{1}$, such that along $\gamma$ the branch $g$ continues analytically to $h$. The path $\gamma$ is homotopic in $\Omega$ to a path $\gamma^{\prime}$ which start and ends at $z_{1}$ and $\gamma^{\prime} \subset H$. Continuation of $g$ along $\gamma^{\prime}$ yields $h$ but the continuation maps $\gamma^{\prime}$ into $F(f)$, and hence remains in $H$. Thus $H$ is completely invariant.

By Theorem 3.1 there are neither Baker domains nor wandering components in $F(f)$. Observe that the orbit of each $a_{j}$ lies in $H$ and has only zero as a limit point. It follows from Remark 1.4 that the only periodic component is $H$. This is the only component of $F(f)$ since any component $H_{1} \subset F(f)$ must map into $H$ by some $f^{n}$. Recall that $H$ is completely invariant.

(ii) Using the Riemann map $\Phi: \mathbb{D}(0, \rho) \rightarrow W$, where $\mathbb{D}(0, \rho) \subset \mathbb{D}(0,1)$ is a disc with $0<\rho<1$ and $W \subset H$ is a domain which contains $\left\{a_{1}, a_{2}, \ldots, a_{p}, 0\right\}$, we shall show that $\bar{W}^{c}$ has a finite set of components $A_{i}, 1 \leq i \leq N$, where each of them is simply connected and bounded.

Let $\Phi$ be the Riemann map from the unit disc $\mathbb{D}$ to $H$ such that $\Phi(0)=0$, and let $\Psi$ be the local inverse of $\Phi$ which is analytic in a neighborhood of zero with $\Psi(0)=0$. Observe that $G=\Psi f \Phi$ is analytic in a neighborhood of zero with $G(0)=0$ and $G^{\prime}(0)=f^{\prime}(0)$. Analytic continuation of $G$ is possible throughout $\mathbb{D}$. Then $G$ is analytic in $\mathbb{D}$ with $G: \mathbb{D} \rightarrow \mathbb{D}$.

Choose $\rho$ such that $0<\rho<1$ and the circumference $C(0, \rho)$ contains none of the countable set of points $\Phi^{-1}\left(a_{j}\right), 1 \leq j \leq p$, while the disc $D(0, \rho)$ contains at least one of $\Phi^{-1}\left(a_{j}\right)$ for every $1 \leq j \leq p$.

The map $\Phi: \mathbb{D}(0, \rho) \rightarrow W$, where $\left\{a_{1}, \ldots, a_{p}, 0\right\} \subset W$, satisfies $f(W) \subset W$ and the boundary $\partial W$ of $W$ is a subset of $\Gamma: \Phi(C(0, \rho))$. The curve $\Gamma$ crosses itself only at points $\Phi(z)$, where $z \in C(0, \rho)$ is such that $T z \in C(0, \rho)$ for some element $T$ of the covering group which is different from the identity.

Claim. There are at most finitely many points where the curve $\Gamma$ crosses itself. 
If this is not the case, there are sequences $z_{n} \in C(0, \rho), z_{n}^{\prime} \in C(0, \rho)$ and $T_{n} \neq I d$ in the covering group such that $z_{n} \rightarrow \alpha, z_{n}^{\prime}=T_{n}\left(z_{n}\right) \rightarrow \beta$. There is a neighborhood $\Delta=D(\alpha, \eta)$ such that $T \Delta \cap \Delta=\emptyset$ for every covering transformation other than the identity. Let $\Delta^{\prime}=D\left(\alpha, \frac{\eta}{2}\right)$ and take $n$ so large that $z_{n}, z_{n+1} \in \Delta^{\prime}$ and $\left|z_{n}^{\prime}-z_{n+1}^{\prime}\right|<\epsilon$, where $\epsilon$ is a number such that $0<4 \epsilon<(1-p) \eta$. Since $T_{n+1}^{-1}$ maps $z_{n+1}^{\prime}$ to $z_{n+1}$ and $D\left(z_{n+1}^{\prime}, 1-\rho\right)$ into the disc $D\left(z_{n+1}, 2\right)$, we have from Schwarz's Lemma that $T_{n+1}^{\prime} z_{n}^{\prime}$ is in $\Delta$, which implies that $T_{n+1}^{-1} T_{n}=I d$. Thus for large $n$ all $T_{n}$ are the same, say $T$. But then $T$ fixes both circles $C(0, \rho)$ and $C(0,1)$ which is possible only if $T(0)=0$, or if $T=I d$ (since $T$ is a covering transformation) which contradicts our assumption about $T_{n}$. Thus $\Gamma$ is an analytic curve with at most a finite set of self-intersections; thus the claim is proved.

Observe that this fact creates loops in $\Gamma$ and each loop must surround at least a pole or an essential singularity, different from $\infty$. If there are no loops in $\Gamma$, the function is transcendental entire, and $H$ is simply connected. In our case the set of self-intersections is not empty.

We have proved that $\bar{W}^{c}$ has a finite set of components $A_{i}, 1 \leq i \leq N$, each of which must be simply connected and bounded by a part of $\Gamma$. Thus $f^{-1}: W^{c} \rightarrow W^{c}$ and for any $n$ the branches of $f^{-n}$ are all analytic in each $A_{i}$ and map $A_{i}$ to disjoints domains $A_{i(n)}$. Since $\Gamma \subset F(f)$, each component of $J(f)$ lies in the interior of some $A_{i(n)}$, for each $n$.

(iii) Finally, we shall prove that all components of the Julia set are singletons.

Assume that some component $\mu$ of $J(f)$ contains more than one point. Now $f^{n}(\mu)$ is in the interior of some $A_{i(n)}$ and we may choose a sequence $n(k)$ such that $n(k) \rightarrow \infty$ as $k \rightarrow \infty$ and each $i(n(k))$ is the same, $A_{1}$ (say). Thus $\mu$ belongs to a component $A^{k}=$ some $A_{1(n(k))}$ of $f^{-n(k)}\left(A_{1}\right)$. The sequence of branches $g_{k}$ of $f^{-n(k)}$, such that $g_{k}\left(A_{1}\right)=A^{k}$, is normal in $A_{1}$. Without loss of generality we may assume that $g_{k}$ is locally uniformly convergent in $A_{1}$ with limit function $h$.

The set $J_{1}=J(f) \cap A_{1}$ is a compact subset of $A_{1}$. Thus if $h$ is constant, we must have diam $g_{k}\left(J_{1}\right) \rightarrow 0$ as $k \rightarrow \infty$ which implies that diam $\mu=0$, against our assumption. Hence $h$ is a non-constant function univalent in $A_{1}$.

Now we take a Jordan curve $\widetilde{\gamma}$ in $A_{1}$ whose interior, denoted by $I(\widetilde{\gamma})$, contains $J_{1}$. Thus $h\left(J_{1}\right)$ is a compact subset of $h(I(\widetilde{\gamma}))$ which is bounded by $h(\widetilde{\gamma})$. For sufficiently large $k, g_{k}(\widetilde{\gamma})$ approximates $h(\widetilde{\gamma})$ closely and there is an open set $U$ such that for all large $k$ we have $\mu \in g_{k}\left(J_{1}\right) \subset U \subset g_{k}(I(\widetilde{\gamma}))$. Thus $f^{n(k)}(U) \subset I(\widetilde{\gamma}) \subset A_{1}$. In particular, all $f^{n(k)}$ and hence all $f^{n}$ are analytic in $U$. Furthermore, for any $n$ there is a greater $n(k)$ so that $n(k)=n+j$ for some $j>0$. Then $f^{n}(U) \subset$ $f^{-j} f^{n(k)}(U) \subset f^{-j}\left(A_{1}\right)$, which is a set disjoint from $W$. It follows that $U \subset F(f)$ which contradicts the fact that $\mu \subset U$. We have proved that all components of $J(f)$ are singletons for $f \in \mathbf{A}$.

Corollary 3.2. Let $f$ be in either class $\mathcal{M} \cap S_{\mathcal{M}}$ or class $\mathcal{K} \cap S_{\mathcal{K}}$ and suppose that there is an attracting fixed point whose Fatou component $H$ contains all the singular values of $f$. Then $J(f)$ is totally disconnected.

Examples of Theorem A. Examples 1 and 2 in Section 2 are examples of Theorem A.

Example 3. Take $f_{\lambda}=\lambda R\left(e^{z}\right)$, where $R(z)=p(z) / q(z)$ and $p(z), q(z)$ are polinomials of degree exactly $n$ without common zeros and such that (a) all the zeros 
of $p(z)$ and $q(z)$ are simples, (b) $q(0) \neq 0$, and (c) $p(1)=0$. Observe that $\infty$ is an essential singularity of $f_{\lambda}$. The set of singular values of $f_{\lambda}$ is finite; thus $f_{\lambda}$ belongs to the class $\mathcal{M} \cap S_{\mathcal{M}}$. If the constant $\lambda$ is suffiently small, Theorem A applies and $f_{\lambda}$ has a totally disconnected Julia set.

Example 4. The family $f_{\lambda, c, \mu}(z)=\lambda e^{\frac{1}{z^{2}-c}}-\mu e^{-c}$ is in class $\mathcal{K} \cap S_{\mathcal{K}}$. The essential singularities are $\pm \sqrt{c}$. When $\lambda=\mu$ the super attracting fixed point is $z=0$ whose Fatou component contains the set of singular values which is finite. By Theorem A the Julia set is totally disconnected.

\section{REFERENCES}

[1] I. N. Baker, Fixpoints and iterates of entire functions, Math. Z. 71 (1959), 146-153. MR.0107015 (21 \#5743)

[2] I. N. Baker, J. Kotus, and Yi Nian Lü, Iterates of meromorphic functions. I, Ergodic Theory Dynam. Systems 11 (1991), no. 2, 241-248, DOI 10.1017/S014338570000612X. MR.1116639 (92m:58113)

[3] I. N. Baker, J. Kotus, and Yi Nian Lü, Iterates of meromorphic functions. II. Examples of wandering domains, J. London Math. Soc. (2) 42 (1990), no. 2, 267-278, DOI 10.1112/jlms/s2-42.2.267. MR1083445 (92m:58114)

[4] I. N. Baker, J. Kotus, and Yi Nian Lü, Iterates of meromorphic functions. III. Preperiodic domains, Ergodic Theory Dynam. Systems 11 (1991), no. 4, 603-618, DOI 10.1017/S0143385700006386. MR1145612(92m:58115)

[5] I. N. Baker, J. Kotus, and Yi Nian Lü, Iterates of meromorphic functions. IV. Critically finite functions, Results Math. 22 (1992), no. 3-4, 651-656, DOI 10.1007/BF03323112. MR1189754 (94c:58166)

[6] I. N. Baker and P. Domínguez, Some connectedness properties of Julia sets, Complex Variables Theory Appl. 41 (2000), no. 4, 371-389. MR1785150 (2001e:30039)

[7] I. N. Baker, P. Domínguez, and M. E. Herring, Dynamics of functions meromorphic outside a small set, Ergodic Theory Dynam. Systems 21 (2001), no. 3, 647-672, DOI 10.1017/S0143385701001328. MR1836425(2002e:37058)

[8] A. Bolsch, Repulsive periodic points of meromorphic functions, Complex Variables Theory Appl. 31 (1996), no. 1, 75-79. MR.1423240 (98c:30033)

[9] A. Bolsch, Iteration of meromorphic functions with countably many singularities. Dissertation, Berlin (1997).

[10] A. Bolsch, Periodic Fatou components of meromorphic functions, Bull. London Math. Soc. 31 (1999), no. 5, 543-555, DOI 10.1112/S0024609399005950. MR.1703869 (2000e:30046)

[11] A. È. Erëmenko and M. Yu. Lyubich, Iterations of entire functions (Russian), Dokl. Akad. Nauk SSSR 279 (1984), no. 1, 25-27. MR769199 (86c:30044)

[12] A. È. Erëmenko and M. Yu. Lyubich, The dynamics of analytic transformations (Russian), Algebra i Analiz 1 (1989), no. 3, 1-70; English transl., Leningrad Math. J. 1 (1990), no. 3, 563-634. MR1015124 (91b:58109)

[13] A. È. Erëmenko and M. Yu. Lyubich, Dynamical properties of some classes of entire functions (English, with English and French summaries), Ann. Inst. Fourier (Grenoble) 42 (1992), no. 4, 989-1020. MR1196102 (93k:30034)

[14] A. L. Epstein, Towers of finite type complex analytic maps, ProQuest LLC, Ann Arbor, MI, 1993. Thesis (Ph.D.), City University of New York. MR 2690048

[15] A.L Epstein, Dynamics of finite type complex analytic maps. I. Global structure theory, Manuscript.

[16] M.E. Herring, An extension of the Julia-Fatou theory of iteration. PhD thesis (1994), Imperial College, London.

[17] P. Fatou, Sur l'itération des fonctions transcendantes Entières (French), Acta Math. 47 (1926), no. 4, 337-370, DOI 10.1007/BF02559517. MR1555220 
[18] L. Keen and J. Kotus, Dynamics of the family $\lambda \tan z$, Conform. Geom. Dyn. 1 (1997), 28-57 (electronic), DOI 10.1090/S1088-4173-97-00017-9. MR1463839 (98h:58159)

[19] L. Rempe, P. J. Rippon, and G. M. Stallard, Are Devaney hairs fast escaping?, J. Difference Equ. Appl. 16 (2010), no. 5-6, 739-762, DOI 10.1080/10236190903282824. MR2675603 (2011i:37061)

F.C. Físico-Matemáticas, Benemérita Universidad Autónoma de Puebla; Av. San Claudio, Col. San Manuel, C.U., Puebla Pue., 72570, México

E-mail address: pdsoto@fcfm.buap.mx

F.C. Físico-Matemáticas, Benemérita Universidad Autónoma de Puebla; Av. San Claudio, Col. San Manuel, C.U., Puebla Pue., 72570, México

E-mail address: hralberto@hotmail.com

Facultad de Ciencias, UnAM. Av. Universidad 3000, C.U. México, D.F., 04510, México.

E-mail address: gsl@dimnamica1.fciencias.unam.mx 